\title{
Characteristics of laparoscopic and open hernia repair simulation models: a systematic review
}

\author{
T. Pelly ${ }^{1}$ (D) J. Vance-Daniel ${ }^{1} \cdot$ C. Linder ${ }^{2}$
}

Received: 14 February 2021 / Accepted: 10 June 2021 / Published online: 2 July 2021

(c) The Author(s), under exclusive licence to Springer-Verlag France SAS, part of Springer Nature 2021

\begin{abstract}
Purpose Barriers to education in open and laparoscopic hernia repair technique include a steep learning curve and reduced theatre time for junior surgical trainees. This is particularly evident during the current COVID-19 pandemic. Simulation models may provide further opportunities for training in hernia repair outside of the traditional surgical apprenticeship model. Methods A systematic review was carried out following PRISMA guidelines to identify and evaluate simulation models in hernia repair. Of the 866 records screened, 27 were included in the analysis. These were assessed for face, content and construct validity, as well as their attempt to measure educational impact.

Results Simulation models were identified comprising of animal tissues, synthetic materials and virtual reality (VR) technology. Models were designed for instruction in repair of inguinal, umbilical, incisional and diaphragmatic hernias. Twenty-one laparoscopic hernia repair models were described. Many models demonstrated validity across several domains, and three showed transferability of skills from simulation to the operating room. Of the six open hernia repair simulation models, none were found to have demonstrated an educational impact in addition to assessing validity.

Conclusion Few models individually were able to demonstrate validity and educational impact. Several novel assessment tools have been developed for assessment of progress when performing simulated and real laparoscopic inguinal hernia repair. More study is required, particularly for open hernia repair, including randomized controlled trials with large sample sizes to assess the transferability of skills.
\end{abstract}

Keywords Hernia $\cdot$ Simulation $\cdot$ Education $\cdot$ Laparoscopy

\section{Introduction}

Hernia repair has long been regarded as an "index" procedure in the early stages of surgical training, and competence in open and laparoscopic hernia repair is viewed by many surgical trainees as a milestone in their careers. While many other surgical procedures are increasingly laparoscopic, open hernia repair continues to be commonly performed. Between 2010 and 2011, the numbers of open inguinal and femoral hernias performed by trainees remained static while open appendicectomy and cholecystectomy fell [1]. Open hernia repair provides opportunities to train in open surgery

T. Pelly

h.pelly@nhs.net

1 Department of General Surgery, St Helier Hospital, London, UK

2 Department of General Surgery, Royal London Hospital, London, UK techniques transferrable to other procedures less frequently performed. However, the COVID-19 pandemic has altered and disrupted surgical training in many ways, with the redeployment of surgical trainees, as well as a reduction in operating workload [2]. As an elective operation, hernia repairs were one of many operations to be cancelled, reducing the opportunities of trainees to learn the procedure.

Concurrently, competence in laparoscopic surgery is becoming increasingly important in surgical training, with a $265 \%$ increase in laparoscopic hernia repairs performed by trainees between 2010 and 2011[1]. Education in laparoscopy is known to be challenging, with one study showing that $79 \%$ of general surgical residents identified a lack of training or a steep learning curve as a barrier to using laparoscopic technique in hernia repair [3]. It is suggested that the disparity between surgeons who regularly perform laparoscopic hernia repair and those who were interested in learning is demonstrative of an educational vacuum. 
Simulation models can be used to help trainees learn procedures in a safe environment while eliminating patient risk. Simulation offers trainees the opportunity to perform procedures that trainees may not be regularly exposed, particularly during the COVID-19 pandemic [4]. The Society of American Gastrointestinal and Endoscopic Surgeons recommend inanimate and animal simulation labs as key components in training for laparoscopic hernia repair [5]. To provide the optimum training environment, simulation models should aim to be realistic, educational and deliver an increase in proficiency on returning to the operating theatre. Following design and implementation of the model, it is essential that developers use robust techniques to assess its educational impact on trainees.

The aims of this systematic review are to describe the current status of simulation in hernia repair surgery and compare these simulation models where possible for their educational value and validity.

\section{Methods}

A systematic review was carried out following the PRISMA organisation guidelines, utilising the PUBMED database. The search included the keywords hernia and simulat* (the use of the wildcard to include derivatives such as simulation/simulate) "hernia AND simulat*" This initial search returned 865 records in December 2020. A further record was identified via the references of an eligible article, bringing the total to 866 . These were reviewed by reviewer TP. Of these 866 records, 40 were felt to be relevant to the aims of the review. References from the reviewed studies were included in the search where appropriate- these are detailed in Fig. 1. Existing reviews relating to simulation models were excluded. Conference abstracts and correspondence letters were also excluded, as they lacked sufficient detail for analysis. On reviewing the full-text articles, a total of 27 articles were eligible for evaluation.

The remaining studies were reviewed by authors using a standardised proforma, classifying simulation models by type-open or laparoscopic, as well as materials used-VR, 3D-printed, synthetic, or animal. Each study was assessed in terms of how thoroughly it was validated, and its performance in any assessment of educational impact. Costs were estimated where these were not documented by the authors, in USD (\$) for ease of comparison. Face validity was defined as the physical realism of the simulator [6]. Construct validity was defined as the ability of the simulator to demonstrate difference in task performance based on experience level [6]. Content validity was defined as the suitability of the simulator as a teaching modality by the formal evaluation of experts [6].

\section{Results}

A total of 27 articles were evaluated in this review. Of these, 21 related to laparoscopic hernia repair simulation models, and 6 were related to open hernia repair. These results are summarised in Table 1 and Table 2.

\section{Laparoscopic hernia repair models}

\section{Totally extra-peritoneal (TEP) inguinal hernia repair}

Few robust attempts at demonstrating educational value are present in the literature regarding simulation models in hernia repair. However, one randomised controlled trial was performed assessing a simulation-based "masterylearning" curriculum for TEP repair against the standard apprenticeship model of surgical training [7]. This study utilised the Guildford MATTU TEP model produced by Limbs and Things LTD in the "mastery-learning" arm. Of the 50 surgical residents who participated, the 26 enrolled in the "mastery-learning" curriculum were found on assessment to perform TEP faster, with higher operative performance scores assessed via the Global Operative Assessment of Laparoscopic Skills (GOALS) tool. This was one of the few trials assessing simulation-based training which demonstrated a positive impact on patient outcomes, with intraoperative and postoperative complications less likely in the "mastery-learning" group. Recurrence rates at 3 months were similar. As discussed by the authors, a limitation of this study is a failure to blind intraoperative assessors. Furthermore, the masterylearning-based curriculum, which places an emphasis on continuing training until "mastery" is achieved rather than training for a fixed duration of time, may not be feasible within the time constraints and pressures of surgical training [8]. As a further study by the authors noted, the time taken for mastery learning was $69 \mathrm{~min}$, comprising two sessions over less than 2 weeks [9]. A randomised control trial (RCT) using this model demonstrated the benefits of part-task training rather than full-task training, in terms of greater cost efficacy and increased retention of skills [10].

Another RCT assessed the effect on a simulation-based model using the McGill Laparoscopic Inguinal Hernia Simulator [11]. 14 surgical residents were randomised to either a simulation-based curriculum, or a control group. The groups were assessed using a specifically developed assessment tool, the Global Operative Assessment of Laparoscopic Skills-Groin Hernia (GOALS-GH). On operating room assessment after 15 days, the simulation-based group was found to have statistically significant improvement in GOALS-GH scores by a mean of 3.4 points, where 
PRISMA Flowchart showing article selection:

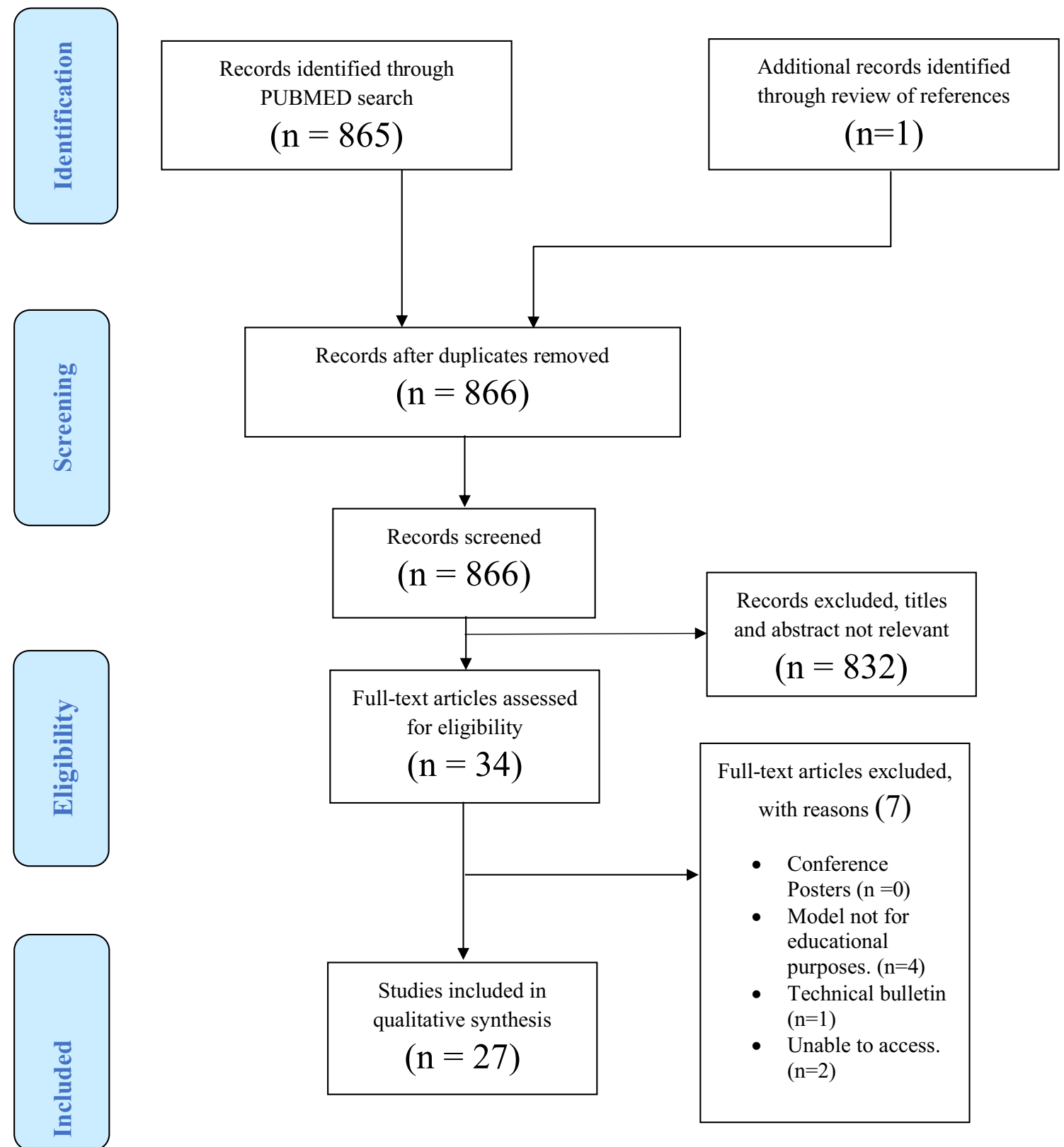

Fig. 1 PRISMA diagram

the control group did not. The sample size was however small and, as the previous RCT, the simulation took place as part of a larger training curriculum and therefore it is different to appreciate the value added by the simulation alone [11].

An elaborate box trainer for TEP inguinal hernia repair was tested on 21 surgical residents, divided into a control and a training group, who were enrolled into an educational programme involving videos and use of the moulded rubber hernia simulator [12]. After the programme, the training groups were found to have higher composite scores on assessment than the control group $(P=0.01)$. Critically, the pre- and post-training assessments were based on observations of the surgeon in the operating theatre, suggesting that the simulator provides transfer of skills into real operative practice. There was, however, no assessment of validity and the model appears very complex to construct.

A low-cost model for TEP laparoscopic inguinal hernia repair was developed by Adrales et al., constructed from an intraoperative photograph enhanced with various cords 
Table 1 Comparison of laparoscopic hernia repair simulation models

\begin{tabular}{|c|c|c|c|c|c|c|c|c|}
\hline \multirow{2}{*}{$\begin{array}{l}\text { Laparoscopic } \\
\text { hernia repair } \\
\text { models } \\
\text { Category }\end{array}$} & \multirow[t]{2}{*}{ Article } & \multirow[t]{2}{*}{ Type of model } & \multicolumn{3}{|c|}{ Validity } & \multirow{2}{*}{$\begin{array}{l}\text { Educa- } \\
\text { tional } \\
\text { impact }\end{array}$} & \multirow{2}{*}{$\begin{array}{l}\text { Transfer } \\
\text { of skills to } \\
\text { OT }\end{array}$} & \multirow[t]{2}{*}{ Type of study } \\
\hline & & & Face & Construct & Content & & & \\
\hline \multirow[t]{8}{*}{ Inguinal TEP } & $\begin{array}{l}\text { Zendejas et al. (Guildford } \\
\text { model) [7] }\end{array}$ & Synthetic & & & & $\checkmark$ & $\checkmark$ & Randomised controlled trial \\
\hline & $\begin{array}{l}\text { Zendejas et al. (Guildford } \\
\text { model) [9] }\end{array}$ & Synthetic & & & & $\checkmark$ & & $\begin{array}{l}\text { Pre-post study with no } \\
\text { control group }\end{array}$ \\
\hline & $\begin{array}{l}\text { Hernandez-Irizarry et al } \\
\text { (Guildford model) [10] }\end{array}$ & Synthetic & & & & $\checkmark$ & & Randomised controlled trial \\
\hline & $\begin{array}{l}\text { Kurashima et al } \\
\text { (McGill model) [11] }\end{array}$ & Synthetic & & & & $\checkmark$ & $\checkmark$ & Randomised controlled trial \\
\hline & Hamilton et al. [12] & Synthetic & & & & $\checkmark$ & & Randomised controlled trial \\
\hline & Adrales et al. [14] & Synthetic & & & & & & Technical description \\
\hline & Adrales et al. [13] & Synthetic & $\checkmark$ & $\checkmark$ & & & & $\begin{array}{l}\text { Technical description with } \\
\text { validity assessment }\end{array}$ \\
\hline & Rowse et al. [15] & Synthetic & & & & $\checkmark$ & & $\begin{array}{l}\text { Pre-post study with no } \\
\text { control group }\end{array}$ \\
\hline \multirow[t]{5}{*}{ TAPP } & Kurashima et al. [16] & Synthetic & $\checkmark$ & & $\checkmark$ & & & $\begin{array}{l}\text { Technical description with } \\
\text { validity assessment }\end{array}$ \\
\hline & Ivakhov et al. [17] & Animal & $\checkmark$ & & $\checkmark$ & & & $\begin{array}{l}\text { Technical description with } \\
\text { validity assessment }\end{array}$ \\
\hline & Nishihara et al. [18] & $3 \mathrm{D}$-printed & & & & & & $\begin{array}{l}\text { Technical description with } \\
\text { validity assessment }\end{array}$ \\
\hline & Nishihara et al. [19] & 3D-printed & & $\checkmark$ & & & & $\begin{array}{l}\text { Technical description with } \\
\text { validity assessment }\end{array}$ \\
\hline & Light et al. [20] & Cadaveric & & & & & $\checkmark$ & Case-control Study \\
\hline \multirow[t]{6}{*}{ Ventral hernia } & $\begin{array}{l}\text { Seagull et al. (SAW } \\
\text { model) [21] }\end{array}$ & Synthetic & & & & & & Technical description \\
\hline & $\begin{array}{l}\text { Vaillancourt et al. (SAW } \\
\text { model) [22] }\end{array}$ & Synthetic & $\checkmark$ & $\checkmark$ & & & & $\begin{array}{l}\text { Validation of assessment } \\
\text { tool }\end{array}$ \\
\hline & $\begin{array}{l}\text { Ghaderi et al. (SAW } \\
\text { model) [23] }\end{array}$ & Synthetic & & $\checkmark$ & & & $\checkmark$ & $\begin{array}{l}\text { Assessment of correlation } \\
\text { with operative perfor- } \\
\text { mance }\end{array}$ \\
\hline & Jain et al. [24] & Synthetic & & & & $\checkmark$ & & $\begin{array}{l}\text { Pre-post study with no } \\
\text { control group }\end{array}$ \\
\hline & Hwang et al. [25] & Synthetic & & & & & & Technical description \\
\hline & Nurczyk et al. [26] & Animal & & & & & & Technical description \\
\hline \multirow[t]{2}{*}{ Paediatric } & Hortense et al. [34] & Synthetic & & & $\checkmark$ & & & $\begin{array}{l}\text { Technical description with } \\
\text { validity assessment }\end{array}$ \\
\hline & Ljuhar et al. [35] & Synthetic & $\checkmark$ & $\checkmark$ & $\checkmark$ & & & $\begin{array}{l}\text { Technical description with } \\
\text { validity assessment }\end{array}$ \\
\hline
\end{tabular}

to represent structures such as the vas deferens [13]. Face validity findings were again inconclusive, as over two-thirds of the participants were year one and two post graduate students. Construct validity however, was demonstrated with participants with more experience performing the procedure faster, without errors, in this unblinded study [14]. Although performance of subjects using this model was assessed, there was no control group or assessment of transferability to real clinical practice, therefore its educational value remains unproven. Another low-fidelity, low-cost model was demonstrated to be an effective modality for teaching inguinal anatomy to a group of 30 general surgery interns [15]. Whether this would translate to an increased ability to recognise anatomical structures intraoperatively remains to be seen, although participants rated the model highly for realism, their prior experience of TEP hernia repair was not documented.

\section{Transabdominal pre-peritoneal (TAPP) hernia repair}

Kurashima et al. developed and validated the McGill Laparoscopic Inguinal Hernia Simulator (M-LIHS), a low-cost 
Table 2 Comparison of open hernia repair models

\begin{tabular}{|c|c|c|c|c|c|c|c|c|}
\hline \multirow{2}{*}{$\begin{array}{l}\text { Open Hernia Repair } \\
\text { models } \\
\text { Location }\end{array}$} & \multirow[t]{2}{*}{ Article } & \multirow[t]{2}{*}{ Type of model } & \multirow[b]{2}{*}{ Face } & \multicolumn{2}{|l|}{ Validity } & \multirow{2}{*}{$\begin{array}{l}\text { Educa- } \\
\text { tional } \\
\text { impact }\end{array}$} & \multirow{2}{*}{$\begin{array}{l}\text { Transfer of } \\
\text { skills to OT }\end{array}$} & \multirow[b]{2}{*}{ Type of study } \\
\hline & & & & Construct & Content & & & \\
\hline Umbilical & Friedrich et al. [27] & Synthetic & $\checkmark$ & $\checkmark$ & $\checkmark$ & $\checkmark$ & & $\begin{array}{l}\text { Pre-post study with no } \\
\text { control group }\end{array}$ \\
\hline Inguinal & Nazari et al. [28] & Synthetic & $\checkmark$ & & $\checkmark$ & & & $\begin{array}{l}\text { Technical description } \\
\text { with validity assess- } \\
\text { ment }\end{array}$ \\
\hline Sub-xiphoid incisional & Zipper et al. [30] & Synthetic & & $\checkmark$ & & & & $\begin{array}{l}\text { Technical description } \\
\text { with validity assess- } \\
\text { ment }\end{array}$ \\
\hline Inguinal & Rosenberg et al. [31] & Animal & & & & & & Technical description \\
\hline Inguinal & Khatib et al. [32] & VR & & & & $\checkmark$ & & $\begin{array}{l}\text { Pre-post study without } \\
\text { control group }\end{array}$ \\
\hline Inguinal & Sanders et al. [33] & VR & & & & & & Technical description \\
\hline
\end{tabular}

adaptable box simulator for evaluation and training of Laparoscopic Inguinal Hernia Repair (LIHR) [16]. Although it is low-cost, it appeared complex to construct from layers of sponge, cling wrap and fabric. Six consultant surgeons who are familiar with laparoscopic inguinal hernia repair were timed and evaluated using the M-LIHS using the GOALS$\mathrm{GH}$. They evaluated the simulator as very useful in training for LIHR $(4.7 \pm 0.5)$, which is suggestive of content validity. However, they considered the simulation to be easier than performing the procedure on a real patient. This is significant, as the model may therefore not be of appropriate for training with more experienced operators, although it may retain some value for novices. Construct validity was not assessed in this study.

Ivakhov et al. describe a box trainer for the simulation of TAPP repair [17]. This composed of a synthetic box structure with a flap or porcine stomach prepared to simulate the abdominal wall. Mechanical testing was used to demonstrate the elastic properties of the gastric mucosa were similar to that of human peritoneum, although it was almost twice as hard to peel from underlying structures. Face and content validity were assessed, with the model rating favourably in terms of realism and usefulness as a tool. However, as the majority $(79 \%)$ of participants had performed $<10$ or no TAPP repair for inguinal hernias before, this assessment may not be reliable. Nishihara et al. developed a 3D-printed physical laparoscopy simulator and handmade organ replica to simulate trans-abdominal pre-peritoneal inguinal hernia repair [18]. This model was later evaluated, and demonstrated to show construct validity [19].

Light et al. compared outcomes of trainees undergoing the Ethicon ${ }^{\mathrm{TM}}$ laparoscopic inguinal groin hernia training (LIGHT) course to the outcomes from a matched control cohort operated on by the consultants leading the course [20]. The course was spread over 3 days, involving simulation practice on a cadaveric model, before performing laparoscopic hernia repair on real patients under direct consultant supervision. The long-term outcomes of the patients who had their operations as part of the course were found to be similar to the matched cohort. This study is reassuring in terms of the outcomes of hernia operations that are performed as part of simulation-based courses, but provides little data to critically analyse the simulation model itself.

\section{Ventral hernia}

Seagull et al. describe a box trainer model for laparoscopic ventral abdominal repair, the Surgical Abdominal Wall (SAW) model [21]. Participants using the simulator reported increased confidence after using the model across a variety of tasks, including sizing and fixation of the mesh. Face and other validity measures were not assessed. Vaillancourt et al. used this same model to develop the Global Operative Assessment of Laparoscopic Skills-Inguinal Hernia (GOALS-IH), a form of Objective Structured Assessment of Technical Skills (OSATS) tool for assessing trainee performance of laparoscopic hernia repair [22]. The mean GOALS-IH score was lower for novices (22.7) than for experts (32.3), suggesting construct validity. To assess for correlation with operating room experience, 14 surgeons were assessed using the GOALS-IH model performing laparoscopic incisional hernia repair both on the SAW model and on actual patients in the operating room [23]. There was good correlation $(0.87)$ between performance in the simulator and the operating room, although it should be noted that novice surgeons achieved lower scores in the operating room, and the study was not able to reach statistical significance in this group.

A similar box training model was designed by Jain et al., which could be used to practice bilateral and unilateral 
inguinal hernia repair (trans-abdominal pre-peritoneal approach), as well as ventral hernia repair [24]. The model was not formally assessed for face, content or construct validity. However, 40 surgeons were assessed using the model as part of a 2-day training course, and were found to have an increase in global and task-specific rating score over the course of the 2 days. There was no assessment of transferability to real practice. Several other models are described, with detailed instructions for producing the models from synthetic materials or porcine flank, without any attempt at validation $[25,26]$.

\section{Open}

In comparison to laparoscopic hernia simulation models, the literature was more scarce for open repair. Only 6 models were described, typically constructed from silicon or animal materials, although two virtual reality models were identified. Costs ranged from between 5 and 109\$.

A silicon-based model of umbilical hernia repair was designed by Friedrich et al. [27]. They used a pre-peritoneal mesh, focusing on haptic feedback via both manual and surgical instrument handling. The model was assessed by 12 novice and 6 expert surgeons, with participants performing the repair using the model once under supervision, and once independently. Face and content validity was assessed using a questionnaire. All participants felt the model was at least acceptable, with the greatest score for realistic handling. There was also very good qualitative feedback from the participants. The construct validity of the model was verified, with experts outperforming beginners. Between the two procedures, novice surgeons improved in all assessed fields. Overall, this model provided an effective representation at relatively low cost with significant teaching value, most notably for novice surgeons.

Nazari et al. also used a low-cost model to simulate Lichtenstein repair of inguinal hernias [28]. Assessment was performed by 66 inexperienced surgical trainees and 10 experienced hernia experts, who rated the model highly in terms of validity. It should be noted that Nazari et al. have used the Rehmann model of simulation validity, evaluating this in context of environmental, equipment and psychological validity [29] - the authors of this review have attempted to correlate these findings with the face, content and construct model. A similar silicon model was produced Zipper et al. used a silicon model to simulate repair of median sub-xiphoidal incisional hernias, providing learners with experience preparing the fatty triangle [30]. Both of these models appear complex to construct, and did not formally assess educational impact. Anaesthetised landrace pigs were used to train surgeons in Lichtenstein mesh repair by Rosenberg et al. [31], although no formal feedback on the model was collected, and the authors recognised that this model will not be feasible for many institutions.

Virtual reality (VR) models of open hernia repair have also been designed. Khatib et al. used a virtual reality model of the inguinal region to facilitate learning of Lichtenstein technique for open mesh repair [32]. This model did not formally assessed validity. In an assessment of 56 medical students, students with teaching involving the model showed improved knowledge of the procedure when compared to the control group. The study is promising that the model improves understanding of the underlying anatomy and procedural steps in medical students and may therefore be a valuable educational tool for novice surgeons. However, notably, the model was not designed for and therefore did not assess any impact on technical skill. Sanders et al. describe the Virtual Reality for Educational Surgical Tools (VREST) platform to simulate open hernia repair using the Lichtenstein method [33], although the authors only discuss the possibilities of the model without assessment of its use.

\section{Paediatric hernia}

In paediatric surgery, Hortense et al. developed a bench model for laparoscopic hernia repair from a plastic bottle, with detailed instructions on its construction [34]. This was assessed by 55 surgeons of varying specialties and grades. There was no formal assessment of face or construct validity, although content validity was assessed in the form of a questionnaire. Participants rated the model favourably, it should be noted however that expert surgeons were less positive in their views towards the model than junior surgeons, suggesting the model is more suitable for trainees in early stages of their careers.

The laparoscopic inguinal and diaphragmatic defect (LIDD) model used by Ljuhar et al. was a novel, low-cost box trainer used to simulate paediatric laparoscopic inguinal hernia repairs and laparoscopic congenital diaphragmatic hernia repair [35]. A statistical significance was shown between the scores of the participants, with the best scores being obtained by consultants, demonstrating good construct validity. Expert surgeons scored LIDD highly for content validity for both inguinal hernia and congenital diaphragmatic hernia repair, and there was also a high level of functional fidelity for both simulated procedures. As with many of the simulation models in this review, further study is needed to assess for transferability to skills to the operating room.

\section{Discussion}

This review has demonstrated the range of simulation models in hernia repair currently reported in the literature. These cover a broad variety of materials (animal, synthetic, 
3D-printed and VR) as well as an array or anatomical locations (epigastric, umbilical, diaphragmatic, incisional and inguinal). In this review, a majority of simulation models were described only in terms of appearance and technical construction, with limited attempts at assessment of educational impact. This highlights the vital need for formal attempts to measure the educational value of the simulation models. The few models that were robustly assessed in terms of validity demonstrated significant improvements operative skills. Most prominently, the SAW model for laparoscopic ventral abdominal repair demonstrated good correlation between scores of both novice and expert surgeons using the simulator and in the operating room. This is a promising finding, although a randomised controlled trial assessing performance would help to confirm this. The Guildford MATTU TEP model also demonstrated superior outcomes to the control group in a randomised controlled trial.

Observed Structured Assessment Tools (OSATS) have are valid frameworks for assessing the skills of surgical trainees in the operating theatre and in a simulationbased setting [36]. The GOALS-GH [37] and GOALS-IH [22] OSATS were designed specifically for TEP and TAPP LIHR and incisional hernia repair, respectively. Both were shown to have high inter-rater reliabilities $(0.96,95 \%$ CI 0.74-0.99) as well as construct validity, with good correlation between GOALS-IH scores and number of laparoscopic procedures performed by the trainee. Future developers of hernia repair models would benefit from employing these assessment tools or similar, to allow for more formal evaluation of models educational impact, as well as greater ease of comparison between simulation models.

There were fewer attempts to measure educational impact in the open hernia repair models than the laparoscopic models. This may be that junior surgical trainees have more access to open hernia repairs, and so the demand for simulation models is lower than for laparoscopic models. However, as non-urgent surgery still has yet to reach normal levels due to the COVID-19 pandemic, the need for these models has increased and is likely to remain so in the near future. Therefore, there is a requirement for good-quality trials to assess the ability for these models to improve trainee's skills and operative room performance.

Overall, this review has demonstrated that the majority of hernia models lack robust attempts at validation and assessment of educational impact. These findings are consistent with similar reviews of surgical simulation models in other fields. The recommendation of the authors of this review is that future research is focused on randomised controlled trials assessing existing promising hernia repair models for their educational value, and transferability of skills to the operating room. Further studies solely describing novel but unvalidated simulation models are unlikely to be of benefit to trainees, or their future patients. Schout et al. described the widespread flaws and variability in surgical simulation methodologies, and outlined a framework for developing more robust methodologies for developing and assessing simulation models [38]. The Consortium of American College of Surgeons-Accredited Education Institutes defined several future directions for research in simulation. Both articles stress the importance of involving educationalists in the development and assessment of simulation programs [39].

This review has a number of limitations. Despite including broad search terms and a high number of records screened for relevance, it is possible that some simulation models of educational value may have been missed. In addition, a single database was searched by a single author, and other reviewers of the identified records may have had different opinions regarding their eligibility. It should be noted that the literature regarding validity is nebulous and indeed the definitions of face, content, and construct validity do vary from the definitions applied in this review. This is particularly true for models which have used a different criteria for validation entirely. As has been previously discussed, there is need for more of a consensus in terms of how validity is assessed in terms of simulation [38].

\section{Conclusion}

There are many hernia simulation models available to individuals or institutions seeking to supplement traditional surgical training. However, there are few attempts to robustly demonstrate the educational impact of simulation models in operation performance. Several models, particularly in laparoscopic hernia repair, can be recommended for use as part of simulation-based curriculum. Further study is needed for open hernia repair simulation models.

Funding Not applicable.

Data availability Not applicable.

Code availability Not applicable.

\section{Declarations}

Conflict of interest The authors declare no conflicts of interest.

Ethical approval Not applicable.

Human and animal rights The authors did not infringe the rights of any humans or animals during the systematic review process. Please see individual articles for further details of their ethical processes.

Informed consent For this type of study formal consent is not required. 


\section{References}

1. McCoy AC et al (2013) Are open abdominal procedures a thing of the past? An analysis of graduating general surgery residents' case logs from 2000 to 2011. J Surg Educ 70(6):683-689

2. James HK, Pattison GTR (2021) Disruption to surgical training during Covid-19 in the United States, United Kingdom, Canada, and Australasia: a rapid review of impact and mitigation efforts. J Surg Educ 78(1):308-314

3. Trevisonno $\mathrm{M}$ et al (2015) A survey of general surgeons regarding laparoscopic inguinal hernia repair: practice patterns, barriers, and educational needs. Hernia 19(5):719-724

4. Davies J, Khatib M, Bello F (2013) Open surgical simulation-a review. J Surg Educ 70(5):618-627

5. Integrating advanced laparoscopy into surgical residency training (1998) Society of American Gastrointestinal Endoscopic Surgeons (SAGES). Surg Endosc 12(4):374-376

6. McDougall EM (2007) Validation of surgical simulators. J Endourol 21(3):244-247

7. Zendejas B et al (2011) Simulation-based mastery learning improves patient outcomes in laparoscopic inguinal hernia repair: a randomized controlled trial. Ann Surg 254(3):502-509 (discussion 509-11)

8. McGaghie WC et al (2014) A critical review of simulationbased mastery learning with translational outcomes. Med Educ 48(4):375-385

9. Zendejas B et al (2012) Mastery learning simulation-based curriculum for laparoscopic TEP inguinal hernia repair. J Surg Educ 69(2):208-214

10. Hernandez-Irizarry R et al (2016) Optimizing training cost-effectiveness of simulation-based laparoscopic inguinal hernia repairs. Am J Surg 211(2):326-335

11. Kurashima $Y$ et al (2014) Simulation-based training improves the operative performance of totally extraperitoneal (TEP) laparoscopic inguinal hernia repair: a prospective randomized controlled trial. Surg Endosc 28(3):783-788

12. Hamilton E, Daniel S, Kapoor A, Nwariaku F, Bergen P, Rege R, Tesfay S, Jones D (2001) Improving operative performance using a laparoscopic hernia simulator. Am J Surg 182:725-728

13. Adrales GL et al (2003) Evaluating minimally invasive surgery training using low-cost mechanical simulations. Surg Endosc 17(4):580-585

14. Adrales GL et al (2003) A valid method of laparoscopic simulation training and competence assessment. J Surg Res 114(2):156-162

15. Rowse PG et al (2016) TEP and Lichtenstein anatomy: does simulation accelerate acquisition among interns? Hernia 20(3):411-416

16. Kurashima $\mathrm{Y}$ et al (2011) A novel low-cost simulator for laparoscopic inguinal hernia repair. Surg Innov 18(2):171-175

17. Ivakhov G et al (2020) Development and evaluation of a novel simulation model for transabdominal preperitoneal (TAPP) inguinal hernia repair. Hernia 24(1):159-166

18. Nishihara Y, Isobe Y, Kitagawa Y (2017) Validation of newly developed physical laparoscopy simulator in transabdominal preperitoneal (TAPP) inguinal hernia repair. Surg Endosc 31(12):5429-5435

19. Nishihara Y, Isobe Y (2021) Preoperative skill evaluation in transabdominal preperitoneal (TAPP) inguinal hernia repair using a three-dimensional printed TAPP repair simulator. Surg Endosc 35(1):270-274

20. Light $\mathrm{D}$ et al (2017) Outcomes of an innovative training course in laparoscopic hernia repair. Ann R Coll Surg Eng1 99(8):614-616

21. Seagull FJ et al (2009) Surgical Abdominal Wall (SAW): a novel simulator for training in ventral hernia repair. Surg Innov 16(4):330-336
22. Vaillancourt $\mathrm{M}$ et al (2011) GOALS-incisional hernia: a valid assessment of simulated laparoscopic incisional hernia repair. Surg Innov 18(1):48-54

23. Ghaderi I et al (2011) Performance of simulated laparoscopic incisional hernia repair correlates with operating room performance. Am J Surg 201(1):40-45

24. Jain M et al (2009) Hernia endotrainer: results of training on selfdesigned hernia trainer box. J Laparoendosc Adv Surg Tech A 19(4):535-540

25. Hwang $\mathbf{J}$ et al (2010) A novel laparoscopic ventral herniorrhaphy training system. Surg Laparosc Endosc Percutan Tech 20(1):e16-e18

26. Nurczyk K et al (2020) A Novel University of North Carolina Laparoscopic Ventral Hernia Repair Simulator. J Laparoendosc Adv Surg Tech A 30(6):608-611

27. Friedrich $U$ et al (2020) Validation and educational impact study of the NANEP high-fidelity simulation model for open preperitoneal mesh repair of umbilical hernia. Hernia 24(4):873-881

28. Nazari $T$ et al (2020) Validity of a low-cost Lichtenstein open inguinal hernia repair simulation model for surgical training. Hernia 24(4):895-901

29. Issenberg SB, Scalese RJ (2008) Simulation in health care education. Perspect Biol Med 51(1):31-46

30. Zipper CT et al (2020) Incisional hernia repair in a high-fidelity silicone model for open retro-muscular mesh implantation with preparation of the fatty triangle: validation and educational impact study. Hernia 24(6):1307-1315

31. Rosenberg $\mathbf{J}$ et al (2013) An animal model to train Lichtenstein inguinal hernia repair. Hernia 17(2):255-258

32. Khatib $\mathrm{M}$ et al (2014) Validation of open inguinal hernia repair simulation model: a randomized controlled educational trial. Am J Surg 208(2):295-301

33. Sanders AJ et al (2006) Validation of open-surgery VR trainer. Stud Health Technol Inform 119:473-476

34. Duboureau H et al (2021) Development and evaluation of a lowcost part-task trainer for laparoscopic repair of inguinal hernia in boys and the acquisition of basic laparoscopy skills. J Pediatr Surg 56(4):674-677

35. Ljuhar D et al (2018) The laparoscopic inguinal and diaphragmatic defect (LIDD) model: a validation study of a novel box trainer model. Surg Endosc 32(12):4813-4819

36. Martin JA et al (1997) Objective structured assessment of technical skill (OSATS) for surgical residents. Br J Surg 84(2):273-278

37. Kurashima $\mathrm{Y}$ et al (2011) A tool for training and evaluation of laparoscopic inguinal hernia repair: the Global Operative Assessment Of Laparoscopic Skills-Groin Hernia (GOALS-GH). Am J Surg 201(1):54-61

38. Schout BM et al (2010) Validation and implementation of surgical simulators: a critical review of present, past, and future. Surg Endosc 24(3):536-546

39. Scott DJ et al (2011) New directions in simulation-based surgical education and training: validation and transfer of surgical skills, use of nonsurgeons as faculty, use of simulation to screen and select surgery residents, and long-term follow-up of learners. Surgery 149(6):735-744

Publisher's Note Springer Nature remains neutral with regard to jurisdictional claims in published maps and institutional affiliations. 